\title{
Generalized bases of finite groups
}

\author{
Benjamin SAmbale(i)
}

\begin{abstract}
Motivated by recent results on the minimal base of a permutation group, we introduce a new local invariant attached to arbitrary finite groups. More precisely, a subset $\Delta$ of a finite group $G$ is called a $p$-base (where $p$ is a prime) if $\langle\Delta\rangle$ is a $p$-group and $\mathrm{C}_{G}(\Delta)$ is $p$-nilpotent. Building on results of Halasi-Maróti, we prove that $p$-solvable groups possess $p$-bases of size 3 for every prime $p$. For other prominent groups, we exhibit $p$-bases of size 2 . In fact, we conjecture the existence of $p$-bases of size 2 for every finite group. Finally, the notion of $p$-bases is generalized to blocks and fusion systems.
\end{abstract}

Mathematics Subject Classification. 20D20, $20 \mathrm{~B} 05$.

Keywords. Base, p-nilpotent centralizer, Fusion.

1. Introduction. Many algorithms in computational group theory depend on the existence of small bases. Here, a base of a permutation group $G$ acting on a set $\Omega$ is a subset $\Delta \subseteq \Omega$ such that the pointwise stabilizer $G_{\Delta}$ is trivial (i.e. if $g \in G$ fixes every $\delta \in \Delta$, then $g=1$ ). The aim of this short note is to introduce a generalized base without the presence of a group action. To this end, let us first consider a finite group $G$ acting faithfully by automorphisms on a $p$-group $P$. If $p$ does not divide $|G|$, then $G$ always admits a base of size 2 by a theorem of Halasi-Podoski [5]. Now suppose that $G$ is $p$-solvable, $P$ is elementary abelian, and $G$ acts completely reducibly on $P$. Then $G$ has a base of size 3 (2 if $p \geq 5$ ) by Halasi-Maróti [4]. In those situations, we may form the semidirect product $H:=P \rtimes G$. Now there exists $\Delta \subseteq P$ such that $|\Delta| \leq 3$ and $\mathrm{C}_{H}(\Delta)=\mathrm{C}_{H}(\langle\Delta\rangle) \leq P$. This motivates the following definition.

Definition 1. Let $G$ be a finite group with Sylow $p$-subgroup $P$. A subset $\Delta \subseteq$ $P$ is called a $p$-base of $G$ if $\mathrm{C}_{G}(\Delta)$ is $p$-nilpotent, i.e. $\mathrm{C}_{G}(\Delta)$ has a normal $p$-complement. 
Clearly, any generating set of $P$ is a $p$-base of $G$ since $\mathrm{C}_{G}(P)=\mathrm{Z}(P) \times$ $\mathrm{O}_{p^{\prime}}\left(\mathrm{C}_{G}(P)\right)$ (this observation is generalized in Lemma 7 below).

Our main theorem extends the work of Halasi-Maróti as follows.

Theorem 2. Every p-solvable group has a p-base of size 3 (2 if $p \geq 5$ ).

Although Halasi-Maróti's theorem does not extend to non- $p$-solvable groups, the situation for $p$-bases seems more fortunate. For instance, if $V$ is a finite vector space in characteristic $p$, then every base of $\mathrm{GL}(V)$ (under the natural action) contains a basis of $V$, so its size is at least $\operatorname{dim} V$. On the other hand, $G=\operatorname{AGL}(V)=V \rtimes \operatorname{GL}(V)$ possesses a $p$-base of size 2 . To see this, let $P$ be the Sylow $p$-subgroup of GL $(V)$ consisting of the upper unitriangular matrices. Let $x \in P$ be a Jordan block of size $\operatorname{dim} V$. Then $\mathrm{C}_{\mathrm{GL}(V)}(x) \leq P \mathrm{Z}(\mathrm{GL}(V))$. For any $y \in \mathrm{C}_{V}(x) \backslash\{1\}$, we obtain a $p$-base $\Delta:=\{x, y\}$ such that $\mathrm{C}_{G}(\Delta) \leq V P$. We have even found a $p$-base consisting of commuting elements. After checking many more cases, we believe that the following might hold.

Conjecture 3. Every finite group has a (commutative) p-base of size 2 for every prime $p$.

The role of the number 2 in Conjecture 3 appears somewhat arbitrary at first. There is, however, an elementary dual theorem: A finite group is $p$ nilpotent if and only if every 2 -generated subgroup is $p$-nilpotent. This can be deduced from the structure of minimal non- $p$-nilpotent groups (see $[6$, Satz IV.5.4]). It is a much deeper theorem of Thompson [8] that the same result holds when " $p$-nilpotent" is replaced by "solvable". Similarly, 2-generated subgroups play a role in the Baer-Suzuki theorem and its variations.

Apart from Theorem 2 we give some more evidence of Conjecture 3.

Theorem 4. Let $G$ be a finite group with Sylow p-subgroup P. Then Conjecture 3 holds for $G$ in the following cases:

(i) $P$ is abelian.

(ii) $G$ is a symmetric group or an alternating group.

(iii) $G$ is a general linear group, a special linear group, or a projective special linear group.

(iv) $G$ is a sporadic simple group or an automorphism group thereof.

Our results on (almost) simple groups carry over to the corresponding quasisimple groups by Lemma 8 below. The notion of $p$-bases generalizes to blocks of finite groups and even to fusion systems.

Definition 5. $\quad$ Let $B$ be a $p$-block of a finite group $G$ with defect group $D$. A subset $\Delta \subseteq D$ is called base of $B$ if $B$ has a nilpotent Brauer correspondent in $\mathrm{C}_{G}(\Delta)$ (see [1, Definition IV.5.38]).

- Let $\mathcal{F}$ be a saturated fusion system on a finite $p$-group $P$. A subset $\Delta \subseteq P$ is called base of $\mathcal{F}$ if there exists a morphism $\varphi$ in $\mathcal{F}$ such that $\varphi(\langle\Delta\rangle)$ is fully $\mathcal{F}$-centralized and the centralizer fusion system $\mathcal{C}:=\mathrm{C}_{\mathcal{F}}(\varphi(\langle\Delta\rangle))$ is trivial, i.e. $\mathcal{C}=\mathcal{F}_{\mathrm{C}_{P}(\Delta)}\left(\mathrm{C}_{P}(\Delta)\right)$ (see [1, Definition I.5.3, Theorem I.5.5]). 
By Brauer's third main theorem, the bases of the principal $p$-block of $G$ are the $p$-bases of $G$ (see [1, Theorem IV.5.9]). Moreover, if $\mathcal{F}$ is the fusion system attached to an arbitrary block $B$, then the bases of $B$ are the bases of $\mathcal{F}$ (see [1, Theorem IV.3.19]). By the existence of exotic fusion systems, the following conjecture strengthens Conjecture 3.

Conjecture 6. Every saturated fusion system has a base of size 2.

We show that Conjecture 6 holds for $p$-groups of order at most $p^{4}$.

\section{Results.}

Proof of Theorem 2. Let $G$ be a $p$-solvable group with Sylow $p$-subgroup $P$. Let $N:=\mathrm{O}_{p^{\prime}}(G)$. For $Q \subseteq P, \mathrm{C}_{G}(Q) N / N$ is contained in $\mathrm{C}_{G / N}(Q N / Q)$. Hence, $\mathrm{C}_{G}(Q)$ is $p$-nilpotent whenever $\mathrm{C}_{G / N}(Q N / Q)$ is $p$-nilpotent. Thus, we may assume that $N=1$. Instead we consider $N:=\mathrm{O}_{p}(G)$. Since $G$ is $p$ solvable, $N \neq 1$. We show by induction on $|N|$ that there exists a $p$-base $\Delta \subseteq N$ such that $\mathrm{C}_{G}(\Delta) \leq N$. By the Hall-Higman lemma (see [6, Hilfssatz VI.6.5]), $\mathrm{C}_{G / N}(N / \Phi(N))=N / \Phi(N)$ where $\Phi(N)$ denotes the Frattini subgroup of $N$. It follows that $\mathrm{O}_{p^{\prime}}(G / \Phi(N))=1$. Hence, by induction, we may assume that $N$ is elementary abelian. Then $\bar{G}:=G / N$ acts faithfully on $N$ and it suffices to find a $p$-base $\Delta \subseteq N$ such that $\mathrm{C}_{\bar{G}}(\Delta)=1$. Thus, we may assume that $G=N \rtimes H$ where $\mathrm{C}_{G}(N)=N$ and $\mathrm{O}_{p}(H)=1$.

Note that $\Phi(G) \leq \mathrm{F}(G)=N$ where $\mathrm{F}(G)$ is the Fitting subgroup of $G$. Since $H$ is contained in a maximal subgroup of $G$, we even have $\Phi(G)<N$. Let $K \unlhd H$ be the kernel of the action of $H$ on $N / \Phi(G)$. By way of contradiction, suppose that $K \neq 1$. Since $K$ is $p$-solvable and $\mathrm{O}_{p}(K) \leq \mathrm{O}_{p}(H)=1$, also $K_{0}:=\mathrm{O}_{p^{\prime}}(K) \neq 1$. Now $K_{0}$ acts coprimely on $N$ and we obtain

$$
N=\left[K_{0}, N\right] \mathrm{C}_{N}\left(K_{0}\right)=\Phi(G) \mathrm{C}_{N}\left(K_{0}\right)
$$

as is well-known. Both $\Phi(G)$ and $\mathrm{C}_{N}\left(K_{0}\right) H$ lie in a maximal subgroup $M$ of $G$. But then $G=N H=\Phi(G) \mathrm{C}_{N}\left(K_{0}\right) H \leq M$, a contradiction. Therefore, $H$ acts faithfully on $N / \Phi(G)$ and we may assume that $\Phi(G)=1$. Then there exist maximal subgroups $M_{1}, \ldots, M_{n}$ of $G$ such that $N_{i}:=M_{i} \cap N<N$ for $i=1, \ldots, n$ and $\bigcap_{i=1}^{n} N_{i}=1$. Since $G=M_{i} N$, the quotients $N / N_{i}$ are simple $\mathbb{F}_{p} H$-modules and $N$ embeds into $N / N_{1} \times \cdots \times N / N_{n}$. Hence, the action of $H$ on $N$ is faithful and completely reducible. Now, by the main result of Halasi-Maróti [4], there exists a $p$-base with the desired properties.

Next we work towards Theorem 4.

Lemma 7. Let $P$ be a Sylow p-subgroup of $G$. Let $Q \unlhd P$ such that $\mathrm{C}_{P}(Q) \leq Q$. Then every generating set of $Q$ is a p-base of $G$.

Proof. Since $P \in \operatorname{Syl}_{p}\left(\mathrm{~N}_{G}(Q)\right)$, we have $\mathrm{Z}(Q)=\mathrm{C}_{P}(Q) \in \operatorname{Syl}_{p}\left(\mathrm{C}_{G}(Q)\right)$ and therefore $\mathrm{C}_{G}(Q)=\mathrm{Z}(Q) \times \mathrm{O}_{p^{\prime}}\left(\mathrm{C}_{G}(Q)\right)$ by the Schur-Zassenhaus theorem.

Lemma 8. Let $\Delta$ be a p-base of $G$ and let $N \leq \mathrm{Z}(G)$. Then $\bar{\Delta}:=\{x N: x \in \Delta\}$ is a p-base of $G / N$. 
Proof. Let $g N \in \mathrm{C}_{G / N}(\bar{\Delta})$. Then $g$ normalizes the nilpotent group $\langle\Delta\rangle N$. Hence, $g$ acts on the unique Sylow $p$-subgroup $P$ of $\langle\Delta\rangle N$. Since $g$ centralizes

$$
\langle\bar{\Delta}\rangle=\langle\Delta\rangle N / N=P N / N \cong P / P \cap N
$$

and $P \cap N \leq N \leq \mathrm{Z}(G), g$ induces a $p$-element in $\operatorname{Aut}(P)$ and also in Aut $(\langle\Delta\rangle N)$. Consequently, there exists a $p$-subgroup $Q \leq \mathrm{N}_{G}(\langle\Delta\rangle N)$ such that $\mathrm{C}_{G / N}(\bar{\Delta})=Q \mathrm{C}_{G}(\Delta N) / N=Q \mathrm{C}_{G}(\Delta) / N$. Since $\mathrm{C}_{G}(\Delta)$ is $p$-nilpotent, so is $Q \mathrm{C}_{G}(\Delta)$ and the claim follows.

The following implies the first part of Theorem 4 .

Proposition 9. Let $P$ be a Sylow p-subgroup of $G$ with nilpotency class $c$. Then $G$ has a p-base of size $2 c$.

Proof. The $p^{\prime}$-group $\mathrm{N}_{G}(\mathrm{Z}(P)) / \mathrm{C}_{G}(\mathrm{Z}(P))$ acts faithfully on $\mathrm{Z}(P)$. By HalasiPodoski [5], there exists $\Delta_{0}=\{x, y\} \subseteq \mathrm{Z}(P)$ such that $\mathrm{N}_{H}(\mathrm{Z}(P)) \leq \mathrm{C}_{H}(\mathrm{Z}(P))$ where $H:=\mathrm{C}_{G}\left(\Delta_{0}\right)$. If $c=1$, then $P=\mathrm{Z}(P)$ is abelian and Burnside's transfer theorem implies that $H$ is $p$-nilpotent. Hence, let $c>1$. By a well-known fusion argument of Burnside, elements of $\mathrm{Z}(P)$ are conjugate in $H$ if and only if they are conjugate in $\mathrm{N}_{H}(\mathrm{Z}(P))$. Consequently, all elements of $\mathrm{Z}(P)$ are isolated in our situation. By the $\mathrm{Z}^{*}$-theorem (assuming the classification of finite simple groups), we obtain

$$
\mathrm{Z}\left(H / \mathrm{O}_{p^{\prime}}(H)\right)=\mathrm{Z}(P) \mathrm{O}_{p^{\prime}}(H) / \mathrm{O}_{p^{\prime}}(H) .
$$

The group $\bar{H}:=H / \mathrm{Z}(P) \mathrm{O}_{p^{\prime}}(H)$ has Sylow $p$-subgroup $\bar{P} \cong P / \mathrm{Z}(P)$ of nilpotency class $c-1$. By induction on $c$, there exists a $p$-base $\overline{\Delta_{1}} \subseteq \bar{P}$ of $\bar{H}$ of size $2(c-1)$. We may choose $\Delta_{1} \subseteq P$ such that $\overline{\Delta_{1}}=\left\{\bar{x}: x \in \Delta_{1}\right\}$. Since $\overline{\mathrm{C}_{H}\left(\Delta_{1}\right)} \leq \mathrm{C}_{\bar{H}}\left(\overline{\Delta_{1}}\right)$ is $p$-nilpotent, so is

$$
\left(\mathrm{C}_{H}\left(\Delta_{1}\right) \mathrm{Z}(P) \mathrm{O}_{p^{\prime}}(H) / \mathrm{O}_{p^{\prime}}(H)\right) / \mathrm{Z}\left(H / \mathrm{O}_{p^{\prime}}(H)\right) .
$$

It follows that $\mathrm{C}_{H}\left(\Delta_{1}\right) \mathrm{Z}(P) \mathrm{O}_{p^{\prime}}(H) / \mathrm{O}_{p^{\prime}}(H)$ and $\mathrm{C}_{H}\left(\Delta_{1}\right)=\mathrm{C}_{G}\left(\Delta_{0} \cup \Delta_{1}\right)$ are $p$-nilpotent as well. Hence, $\Delta:=\Delta_{0} \cup \Delta_{1}$ is a $p$-base of $G$ of size (at most) $2 c$.

Proposition 10. The symmetric and alternating groups $S_{n}$ and $A_{n}$ have commutative $p$-bases of size 2 for every prime $p$.

Proof. Let $n=\sum_{i=0}^{k} a_{i} p^{i}$ be the $p$-adic expansion of $n$. Suppose first that $G=S_{n}$. Let

$$
x=\prod_{i=0}^{k} \prod_{j=1}^{a_{i}} x_{i j} \in G
$$

be a product of disjoint cycles $x_{i j}$ where $x_{i j}$ has length $p^{i}$ for $j=1, \ldots, a_{i}$. Then $x$ is a $p$-element and

$$
\mathrm{C}_{G}(x) \cong \prod_{i=0}^{k} C_{p^{i}}\left\langle S_{a_{i}} .\right.
$$


Since $a_{i}\left\langle p, P:=\left\langle x_{i j}: i=0, \ldots, k, j=1, \ldots, a_{i}\right\rangle\right.$ is an abelian Sylow $p$-subgroup of $\mathrm{C}_{G}(x)$. Let $y:=\prod_{i=0}^{k} \prod_{j=1}^{a_{i}} x_{i j}^{j} \in P$. It is easy to see that $\Delta:=\{x, y\}$ is a commutative $p$-base of $G$ with $\mathrm{C}_{G}(\Delta)=P$.

Now let $G=A_{n}$. If $p>2$, then $x, y$ lie in $A_{n}$ as constructed above and the claim follows from $\mathrm{C}_{A_{n}}(\Delta) \leq \mathrm{C}_{S_{n}}(\Delta)$. Hence, let $p=2$. If $\sum_{i=1}^{k} a_{i} \equiv 0$ $(\bmod 2)$, then we still have $x \in A_{n}$ and $\mathrm{C}_{G}(x)=\left\langle x_{i j}: i, j\right\rangle$ is already a 2group. Thus, we have a 2-base of size 1 in this case. In the remaining case, let $m \geq 1$ be minimal such that $a_{m}=1$. We adjust our definition of $x$ by replacing $x_{m 1}$ with a disjoint product of two cycles of length $2^{m-1}$. Then $x \in A_{n}$ and $\mathrm{C}_{G}(x)$ is a 2-group or a direct product of a 2-group and $S_{3}$ (the latter case happens if and only if $\left.m=1=a_{0}\right)$. We clearly find a 2-element $y \in \mathrm{C}_{G}(x)$ such that $\mathrm{C}_{G}(x, y)$ is a 2 -group.

The following elementary facts are well-known, but we provide proofs for the convenience of the reader.

Lemma 11. Let $p$ be a prime and let $q$ be a prime power such that $p \nmid q$. Let $e \mid p-1$ be the multiplicative order of $q$ modulo $p$. Let $p^{s}$ be the p-part of $q^{e}-1$. Then for every $n \geq 1$, the polynomial $X^{p^{n}}-1$ decomposes as

$$
X^{p^{n}}-1=(X-1) \prod_{k=1}^{\left(p^{s}-1\right) / e} \gamma_{0, k} \prod_{i=1}^{n-s} \prod_{k=1}^{\varphi\left(p^{s}\right) / e} \gamma_{i, k}
$$

where the $\gamma_{i, k}$ are pairwise coprime polynomials in $\mathbb{F}_{q}[X]$ of degree ep $p^{i}$ for $i=0, \ldots, n-s$.

Proof. Let $\zeta$ be a primitive root of $X^{p^{n}}-1$ in some finite field extension of $\mathbb{F}_{q}$. Then

$$
X^{p^{n}}-1=\prod_{k=0}^{p^{n}-1}\left(X-\zeta^{k}\right) .
$$

Recall that $\mathbb{F}_{q}$ is the fixed field under the Frobenius automorphism $c \mapsto c^{q}$. Hence, the irreducible divisors of $X^{p^{n}}-1$ in $\mathbb{F}_{q}[X]$ correspond to the orbits of $\left\langle q+p^{n} \mathbb{Z}\right\rangle$ on $\mathbb{Z} / p^{n} \mathbb{Z}$ via multiplication. The trivial orbit corresponds to $X-1$. For $i=1, \ldots, s$, the order of $q$ modulo $p^{i}$ is $e$ by the definition of $s$. This yields $\left(p^{s}-1\right) / e$ non-trivial orbits of length $e$ in $p^{n-s} \mathbb{Z} / p^{n} \mathbb{Z}$. The corresponding irreducible factors are denoted by $\gamma_{0, k}$ for $k=1, \ldots,\left(p^{s}-1\right) / e$.

For $i \geq 1$, the order of $q$ modulo $p^{s+i}$ divides $e p^{i}$ (it can be smaller if $p=2$ and $s=1)$. We partition $\left(p^{n-s-i} \mathbb{Z} / p^{n} \mathbb{Z}\right)^{\times}$into $\varphi\left(p^{s+i}\right) /\left(e p^{i}\right)=\varphi\left(p^{s}\right) / e$ unions of orbits under $\left\langle q+p^{n} \mathbb{Z}\right\rangle$ such that each union has size $e p^{i}$. The corresponding polynomials $\gamma_{i, 1}, \ldots, \gamma_{i, \varphi\left(p^{s}\right) / e}$ are pairwise coprime (but not necessarily irreducible).

Lemma 12. Let $A$ be an $n \times n$-matrix over an arbitrary field $F$ such that the minimal polynomial of $A$ has degree $n$. Then every matrix commuting with $A$ is a polynomial in $A$. 
Proof. By hypothesis, $A$ is similar to a companion matrix. Hence, there exists a vector $v \in F^{n}$ such that $\left\{v, A v, \ldots, A^{n-1} v\right\}$ is a basis of $F^{n}$. Let $B \in F^{n \times n}$ such that $A B=B A$. There exist $a_{0}, \ldots, a_{n-1} \in F$ such that $B v=a_{0} v+\cdots+$ $a_{n-1} A^{n-1} v$. Set $\gamma:=a_{0}+a_{1} X+\cdots+a_{n-1} X^{n-1}$. Then

$$
B A^{i} v=A^{i} B v=a_{0} A^{i} v+\cdots+a_{n-1} A^{n-1} A^{i} v=\gamma(A) A^{i} v
$$

for $i=0, \ldots, n-1$. Since $\left\{v, A v, \ldots, A^{n-1} v\right\}$ is a basis, we obtain $B=\gamma(A)$ as desired.

Proposition 13. The groups $\operatorname{GL}(n, q), \operatorname{SL}(n, q)$, and $\operatorname{PSL}(n, q)$ possess commutative $p$-bases of size 2 for every prime $p$.

Proof. Let $q$ be a prime power. By Lemma 8 , it suffices to consider GL $(n, q)$ and $\operatorname{SL}(n, q)$. Suppose first that $p \mid q$. Let $x \in G:=\operatorname{GL}(n, q)$ be a Jordan block of size $n \times n$ with eigenvalue 1 . Then $x$ is a $p$-element since $x^{p^{n}}-1=(x-1)^{p^{n}}=0$. Moreover, $\mathrm{C}_{G}(x)$ consists of polynomials in $x$ by Lemma 12. In particular, $\mathrm{C}_{G}(x)$ is abelian and therefore $p$-nilpotent. Hence, we found a $p$-base of size 1. Since $(q-1, p)=1$, this is also a $p$-base of $\operatorname{SL}(n, q)$.

Now let $p \nmid q$. We "linearize" the argument from Proposition 10. Let $e$ and $s$ be as in Lemma 11. Let $0 \leq a_{0} \leq e-1$ be such that $n \equiv a_{0}(\bmod e)$. Let

$$
\frac{n-a_{0}}{e}=\sum_{i=0}^{r} a_{i+1} p^{i}
$$

be the $p$-adic expansion. Let $M_{i} \in \mathrm{GL}\left(e p^{i}, q\right)$ be the companion matrix of the polynomial $\gamma_{i, 1}$ from Lemma 11 for $i=0, \ldots, r$. Let $G_{i}:=\operatorname{GL}\left(e a_{i+1} p^{i}, q\right)$ and $x_{i}:=\operatorname{diag}\left(M_{i}, \ldots, M_{i}\right) \in G_{i}$. Then the minimal polynomial of

$$
x:=\operatorname{diag}\left(1_{a_{0}}, x_{0}, \ldots, x_{r}\right) \in G
$$

divides $X^{p^{r+s}}-1$ by Lemma 11. In particular, $x$ is a $p$-element. Since the $\gamma_{i, 1}$ are pairwise coprime, it follows that

$$
\mathrm{C}_{G}(x)=\mathrm{GL}\left(a_{0}, q\right) \times \prod_{i=0}^{r} \mathrm{C}_{G_{i}}\left(x_{i}\right) .
$$

Since $a_{0}<e, \operatorname{GL}\left(a_{0}, q\right)$ is a $p^{\prime}$-group. By Lemma 12 , every matrix commuting with $M_{i}$ is a polynomial in $M_{i}$. Hence, the elements of $\mathrm{C}_{G_{i}}\left(x_{i}\right)$ have the form $A=\left(A_{k l}\right)_{1 \leq k, l \leq a_{i+1}}$ where each block $A_{k l}$ is a polynomial in $M_{i}$. We define

$$
y_{i}:=\operatorname{diag}\left(M_{i}, M_{i}^{2}, \ldots, M_{i}^{a_{i+1}}\right) \in \mathrm{C}_{G_{i}}\left(x_{i}\right)
$$

and $y:=\operatorname{diag}\left(1_{a_{0}}, y_{0}, \ldots, y_{r}\right) \in \mathrm{C}_{G}(x)$. Let $A=\left(A_{k l}\right) \in \mathrm{C}_{G_{i}}\left(x_{i}, y_{i}\right)$. We want to show that $A_{k l}=0$ for $k \neq l$. To this end, we may assume that $k<l$ and $A_{k l}=\rho\left(M_{i}\right)$ where $\rho \in \mathbb{F}_{q}[X]$ with $\operatorname{deg}(\rho)<\operatorname{deg}\left(\gamma_{i, 1}\right)=e p^{i}$. Since $A \in \mathrm{C}_{G_{i}}\left(x_{i}, y_{i}\right)$, we have $M_{i}^{k} A_{k l}=M_{i}^{l} A_{k l}$ and $\left(M^{l-k}-1\right) A_{k l}=0$. It follows that the minimal polynomial $\gamma_{i, 1}$ of $M_{i}$ divides $\left(X^{l-k}-1\right) \rho$. By way of contradiction, we assume that $\rho \neq 0$. Then $\gamma_{i, 1}$ divides $X^{l-k}-1$ and $X^{p^{r+s}}-1$. However, $l-k \leq a_{i+1}<p$ and $\gamma_{i 1}$ must divide $X-1$. This contradicts the 
definition of $\gamma_{i, 1}$ in Lemma 11. Hence, $A_{k l}=0$ for $k \neq l$. We have shown that the elements of $\mathrm{C}_{G}(x, y)$ have the form

$$
L \oplus \bigoplus_{i=0}^{r} \bigoplus_{j=1}^{a_{i+1}} L_{i j}
$$

where $L \in \mathrm{GL}\left(a_{0}, q\right)$ and each $L_{i j}$ is a polynomial in $M_{i}$. In particular, $\mathrm{C}_{G}(x, y)$ is a direct product of a $p^{\prime}$-group and an abelian group. Consequently, $\mathrm{C}_{G}(x, y)$ is $p$-nilpotent.

Now let $G:=\operatorname{SL}(n, q)$. If $p \nmid q-1$, then the $p$-base of $\operatorname{GL}(n, q)$ constructed above already lies in $G$. Thus, we may assume that $p \mid q-1$. Then $e=1$ and $a_{0}=0$ with the notation above. We now have the polynomials $\gamma_{i, k}$ with $i=0, \ldots, r$ and $k=1, \ldots, p-1 \leq \varphi\left(p^{s}\right)$ at our disposal. Let $M_{i, k}$ be the companion matrix of $\gamma_{i, k}$. Define

$$
x_{i}:=\operatorname{diag}\left(M_{i, 1}, \ldots, M_{i, a_{i+1}}\right)
$$

for $i=0, \ldots, r$. Then the minimal polynomial of $x:=\operatorname{diag}\left(x_{0}, \ldots, x_{r}\right) \in$ $\mathrm{GL}(n, q)$ has degree $n$ and therefore $\mathrm{C}_{\mathrm{GL}(n, q)}(x)$ is abelian by Lemma 12. Let $i \geq 0$ be minimal such that $a_{i+1}>0$. We replace the block $M_{i, 1}$ of $x$ by the companion matrix of $X^{p^{i}}-1$. Then, by Lemma 11, the minimal polynomial of $x$ still has degree $n$. Moreover, $x$ has at least one block $B$ of size $1 \times 1$. We may modify $B$ such that $\operatorname{det}(x)=1$. After doing so, it may happen that $B$ occurs twice in $x$. In this case, $\mathrm{C}_{G}(x) \leq \mathrm{GL}(2, q) \times H$ where $H$ is abelian. Then the matrix

$$
y:= \begin{cases}\left(\begin{array}{cc}
0 & -1 \\
1 & 0
\end{array}\right) \oplus 1_{n-2} & \text { if } p=2, \\
\operatorname{diag}\left(M_{0,1}, M_{0,1}^{-1}, 1_{n-2}\right) & \text { if } p>2\end{cases}
$$

lies in $\mathrm{C}_{G}(x)$ and $\mathrm{C}_{G}(x, y)$ is abelian. Hence, $\{x, y\}$ is a $p$-base of $G$.

Proposition 13 can probably be generalized to classical groups. The next result completes the proof of Theorem 4.

Proposition 14. Let $S$ be a sporadic simple group and $G \in\{S, S .2\}$. Then $G$ has a commutative $p$-base of size 2 for every prime $p$.

Proof. If $p^{4}$ does not divide $|G|$, then the claim follows from Lemma 7 . So we may assume that $p^{4}$ divides $|G|$. From the character tables in the Atlas [2], we often find $p$-elements $x \in G$ such that $\mathrm{C}_{G}(x)$ is already a $p$-group. In this case, we found a $p$-base of size 1 and we are done. If $G$ admits a permutation representation of "moderate" degree (including $\mathrm{Co}_{1}$ ), then the claim can be shown directly in GAP [3]. In the remaining cases, we use the Atlas to find $p$-elements with small centralizers:

- $G=L y, p=2$ : There exists an involution $x \in G$ such that $\mathrm{C}_{G}(x)=$ 2. $A_{11}$. By the proof of Proposition 10, there exists $y \in A_{11}$ such that $\mathrm{C}_{A_{11}}(y)$ is a 2-group. We identify $y$ with a preimage in $\mathrm{C}_{G}(x)$. Then $\mathrm{C}_{G}(x, y)$ is a 2-group. 
- $G=L y, p=3$ : Here we find $x \in G$ of order 3 such that $\mathrm{C}_{G}(x)=3 . M c L$. Since $M c L$ contains a 3 -element $y$ such that $\mathrm{C}_{M c L}(y)$ is a 3 -group, the claim follows.

- $G=T h, p=2$ : There exists an involution $x \in G$ such that $\mathrm{C}_{G}(x)=$ $2_{+}^{1+8} \cdot A_{9}$. As before, we find $y \in \mathrm{C}_{G}(x)$ such that $\mathrm{C}_{G}(x, y)$ is a 2-group.

- $G=M, p=5$ : There exists a 5-element $x \in G$ such that $\mathrm{C}_{G}(x)=$ $C_{5} \times H N$. Since there is also a 5-element $y \in H N$ such that $\mathrm{C}_{H N}(y)$ is a 5-group, the claim follows.

- $G=M, p=7$ : In this case there exists a radical subgroup $Q \leq G$ such that $\mathrm{C}_{G}(Q)=Q \cong C_{7} \times C_{7}$ by Wilson [9, Theorem 7] (this group was missing in the list of local subgroups in the Atlas). Any generating set of $Q$ of size 2 is a desired $p$-base of $G$.

- $G=H N .2, p=3$ : There exists an element $x \in G$ of order 9 such that $\left|\mathrm{C}_{G}(x)\right|=54$. Clearly, we find $y \in \mathrm{C}_{G}(x)$ such that $\mathrm{C}_{G}(x, y)$ is 3-nilpotent.

Finally, we consider a special case of Conjecture 6 .

Proposition 15. Let $\mathcal{F}$ be a saturated fusion system on a p-group $P$ of order at most $p^{4}$. Then $\mathcal{F}$ has a base of size 2 .

Proof. Recall that $A:=\operatorname{Out}_{\mathcal{F}}(P)$ is a $p^{\prime}$-group and there is a well-defined action of $A$ on $P$ by the Schur-Zassenhaus theorem. If $\mathcal{F}$ is the fusion system of the group $P \rtimes A$, then the claim follows from Halasi-Podoski [5] as before. We may therefore assume that $P$ contains an $\mathcal{F}$-essential subgroup. In particular, $P$ is non-abelian. Let $Q<P$ be a maximal subgroup of $P$ containing $\mathrm{Z}(P)$. The fusion system $\mathrm{C}_{\mathcal{F}}(Q)$ on $\mathrm{C}_{P}(Q)=\mathrm{Z}(Q)$ is trivial by definition. Hence, we are done whenever $Q$ is generated by two elements.

It remains to deal with the case where $|P|=p^{4}$ and all maximal subgroups containing $\mathrm{Z}(P)$ are elementary abelian of rank 3 . Since two such maximal subgroups intersect in $\mathrm{Z}(P)$, we obtain that $|\mathrm{Z}(P)|=p^{2}$ and $\left|P^{\prime}\right|=p$ by [7, Lemma 1.9] for instance. By the first part of the proof, we may choose an $\mathcal{F}$-essential subgroup $Q$ such that $\mathrm{Z}(P)<Q<P$. Let $A:=\operatorname{Aut}_{\mathcal{F}}(Q)$. Since $Q$ is essential, $P / Q$ is a non-normal Sylow $p$-subgroup of $A$ (see $[1$, Proposition I.2.5]). Moreover, $[P, Q]=P^{\prime}$ has order $p$. By [7, Lemma 1.11], there exists an $A$-invariant decomposition

$$
Q=\langle x, y\rangle \times\langle z\rangle \text {. }
$$

We may choose those elements such that $\Delta:=\{x z, y\} \nsubseteq \mathrm{Z}(P)$. Then $\mathrm{C}_{P}(\Delta)=$ $Q$ and $\mathrm{C}_{A}(\Delta)=1$. Let $\varphi: S \rightarrow T$ be a morphism in $\mathcal{C}:=\mathrm{C}_{\mathcal{F}}(\Delta)$ where $S, T \leq Q$. Then $\varphi$ extends to a morphism $\hat{\varphi}: S\langle\Delta\rangle \rightarrow T\langle\Delta\rangle$ in $\mathcal{F}$ such that $\hat{\varphi}(x)=x$ for all $x \in\langle\Delta\rangle$. Hence, if $S \leq\langle\Delta\rangle$, then $\varphi=$ id. Otherwise, $S\langle\Delta\rangle=Q$ and $\hat{\varphi} \in \mathrm{C}_{A}(\Delta)=1$ since morphisms are always injective. In any case, $\mathcal{C}$ is the trivial fusion system and $\Delta$ is a base of $\mathcal{F}$.

Acknowledgements. The author is supported by the German Research Foundation (SA 2864/1-2 and SA 2864/3-1).

Funding Open Access funding enabled and organized by Projekt DEAL. 
Open Access. This article is licensed under a Creative Commons Attribution 4.0 International License, which permits use, sharing, adaptation, distribution and reproduction in any medium or format, as long as you give appropriate credit to the original author(s) and the source, provide a link to the Creative Commons licence, and indicate if changes were made. The images or other third party material in this article are included in the article's Creative Commons licence, unless indicated otherwise in a credit line to the material. If material is not included in the article's Creative Commons licence and your intended use is not permitted by statutory regulation or exceeds the permitted use, you will need to obtain permission directly from the copyright holder. To view a copy of this licence, visit http://creativecommons. org/licenses/by/4.0/.

Publisher's Note Springer Nature remains neutral with regard to jurisdictional claims in published maps and institutional affiliations.

\section{References}

[1] Aschbacher, M., Kessar, R., Oliver, B.: Fusion Systems in Algebra and Topology. London Mathematical Society Lecture Note Series, vol. 391. Cambridge University Press, Cambridge (2011)

[2] Conway, J.H., Curtis, R.T., Norton, S.P., Parker, R.A., Wilson, R.A.: ATLAS of Finite Groups. Oxford University Press, Eynsham (1985)

[3] The GAP Group, GAP: Groups, Algorithms, and Programming, Version 4.11.0 (2020). http://www.gap-system.org

[4] Halasi, Z., Maróti, A.: The minimal base size for a $p$-solvable linear group. Proc. Amer. Math. Soc. 144, 3231-3242 (2016)

[5] Halasi, Z., Podoski, K.: Every coprime linear group admits a base of size two. Trans. Amer. Math. Soc. 368, 5857-5887 (2016)

[6] Huppert, B.: Endliche Gruppen. I. Grundlehren der Mathematischen Wissenschaften, vol. 134. Springer, Berlin (1967)

[7] Oliver, B.: Simple fusion systems over $p$-groups with abelian subgroup of index p: I. J. Algebra 398, 527-541 (2014)

[8] Thompson, J.G.: Nonsolvable finite groups all of whose local subgroups are solvable. Bull. Amer. Math. Soc. 74, 383-437 (1968)

[9] Wilson, R.A.: The odd-local subgroups of the Monster. J. Austral. Math. Soc. Ser. A 44, 1-16 (1988)

Benjamin Sambale

Institut für Algebra, Zahlentheorie und Diskrete Mathematik

Leibniz Universität Hannover

Welfengarten 1

30167 Hannover

Germany

e-mail: sambale@math.uni-hannover.de 
Received: 4 September 2020

Revised: 13 January 2021

Accepted: 28 January 2021. 